\title{
ASYMPTOTIC EXPANSIONS OF RATIOS OF COEFFICIENTS OF ORTHOGONAL POLYNOMIALS WITH EXPONENTIAL WEIGHTS
}

\author{
BY \\ ATTILA MÁTE, PAUL NEVAI AND THOMAS ZASLAVSKY ${ }^{1}$
}

\begin{abstract}
Let $p_{n}(x)=\gamma_{n} x^{n}+\cdots$ denote the $n$th polynomial orthonormal with respect to the weight $\exp \left(-x^{\beta} / \beta\right)$ where $\beta>0$ is an even integer. G. Freud conjectured and Al. Magnus proved that, writing $a_{n}=\gamma_{n-1} / \gamma_{n}$, the expression $a_{n} n^{-1 / \beta}$ has a limit as $n \rightarrow \infty$. It is shown that this expression has an asymptotic expansion in terms of negative even powers of $n$. In the course of this, a combinatorial enumeration problem concerning one-dimensional lattice walk is solved and its relationship to a combinatorial identity of J. L. W. V. Jensen is explored.
\end{abstract}

Consider the polynomials $p_{n}$ that are orthonormal with respect to the weight function $\exp \left(-|x|^{\beta} / \beta\right)$ on the real line, where $\beta$ is a positive real number. Denoting by $\gamma_{n}$ the leading coefficient of $p_{n}(n \geqslant 0)$ and writing $a_{n}=\gamma_{n-1} / \gamma_{n}$ for $n \geqslant 1$ and $a_{n}=0$ for $n \leqslant 0$, G. Freud conjectured that

$$
\lim _{n \rightarrow \infty} a_{n} / n^{1 / \beta}=\left(\begin{array}{c}
\beta-1 \\
(\beta-2) / 2
\end{array}\right)^{-1 / \beta}
$$

holds for every positive even $\beta$ (see [3, Conjecture, p. 5]; his conjecture has a slightly different form, as he considered the weight function $|x|^{\rho} \exp \left(-|x|^{\beta}\right)$ rather than the one above). He also entertained the possibility that this conjecture is valid for all positive real $\beta$. In case $\beta>0$ is even, he proved that if the limit on the left exists then it must have the value on the right-hand side (see [3, Theorem 1 on p. 4]), and he established the conjecture for $\beta=2,4$, and 6 (see [3, pp. 5-6]). He accomplished these by extracting information from the formula

$$
\frac{n}{a_{n}}=\int_{-\infty}^{\infty} p_{n}(x) p_{n-1}(x) x^{-1}|x|^{\beta} \exp \left(-|x|^{\beta} / \beta\right) d x
$$

Received by the editors October 30, 1983.

1980 Mathematics Subject Classification. Primary 42C05; Secondary 05A15, 05A19, 41A60.

Key words and phrases. Asymptotic expansion, combinatorial enumerations, combinatorial identities, Freud's conjecture, Jensen's identity, orthogonal polynomials.

${ }^{1}$ This material is based upon work supported by the National Science Foundation under Grant Nos. MCS 8100673 (first author) and MCS-83-00882 (second author) and by the PSC-CUNY Research Award Program of the City University of New York under Grant No. 662043 (first author). The first author made his contribution to this work partly during his stay at The Institute for Advanced Study, Princeton, New Jersey, in the summer of 1983. 
valid for all $n>0$, which he obtained in a somewhat more general setting in [ 2 , Lemma 1, p. 93]. This formula is established via integrating by parts the right-hand side of the equation

$$
\begin{aligned}
\frac{n}{a_{n}} & =\int_{-\infty}^{\infty}\left(n \gamma_{n} x^{n-1}\right) p_{n-1}(x) \exp \left(-|x|^{\beta} / \beta\right) d x \\
& =\int_{-\infty}^{\infty} p_{n}^{\prime}(x)\left(p_{n-1}(x) \exp \left(-|x|^{\beta} / \beta\right)\right) d x .
\end{aligned}
$$

Conjecture (1) for positive even values of $\beta$ was established by Al. Magnus in December 1983; his proof will appear in [19]. Previous important works related to Freud's conjecture are Mhaskar-Saff [12] and Rahmanov [16]. In particular, they establish the weaker result

$$
\lim _{n \rightarrow \infty} n^{-1 / \beta}\left(\prod_{j=1}^{n} a_{j}\right)^{1 / n}=\lim _{n \rightarrow \infty} \gamma_{n}^{-1 / n} n^{-1 / \beta}=\left(\begin{array}{c}
\beta-1 \\
(\beta-2) / 2
\end{array}\right)^{-1 / \beta}
$$

holds for every real $\beta>0$ (cf. [12, formula (3.11) in Theorem 3.3] and the last formula of Theorem 1 in $[16, \S 4$, p. 183]).

The aim of the present paper is to extract additional information from (2) so as to establish

THEOREM 1. Let $\beta>0$ be an even integer. Then $a_{n} / n^{1 / \beta}$ has an asymptotic expansion

$$
a_{n} / n^{1 / \beta} \sim \sum_{l=0}^{\infty} c_{2 l} n^{-2 l}
$$

In other words,

$$
a_{n} / n^{1 / \beta}=\sum_{l=0}^{m} c_{2 l} n^{-2 l}+o\left(n^{-2 m}\right)
$$

for every integer $m \geqslant 0$ as $n \rightarrow \infty$.

A detailed discussion of asymptotic expansions can be found e.g. in P. Henrici [6, Chapter 11, pp. $351 \mathrm{ff}]$. We do not know whether or not there is a value of $n$ for which the series on the right of (3) converges. Theorem 1 is known in the special cases $\beta=2,4,6$, which are exactly the cases in which Freud [3, pp. 5-6] established the validity of (1). In case $\beta=2$, the $p_{n}(x / \sqrt{2})$ are the Hermite polynomials (save for a constant factor depending on $n$ ), and it is easy to see that $a_{n}=\sqrt{n}$ (cf. (8) below). For $\beta=4$, Theorem 1 was proved by J. S. Lew and D. A. Quarles, Jr. [10], and for $\beta=6$, it was established in Máté-Nevai [11]. The polynomials $p_{n}$ for $\beta=4$ were discussed by Nevai in [14 and 15]. The latter paper uses the asymptotic expansion given in (3) to derive asymptotic properties of the polynomials $p_{n}$ in case $\beta=4$.

From now on throughout this paper, we will assume that $\beta$ is a positive even integer, and we will write

$$
\beta=2 k+2 \quad(k \geqslant 0) .
$$


Then (2) becomes

$$
\frac{n}{a_{n}}=\int_{-\infty}^{\infty} x^{2 k+1} p_{n}(x) p_{n-1}(x) \exp \left(-x^{2 k+2} /(2 k+2)\right) d x
$$

which is easier to handle than (2) is in general because of the absence of the absolute value signs. By using the recurrence formula

$$
x p_{n}(x)=a_{n+1} p_{n+1}(x)+a_{n} p_{n-1}(x) \quad(-\infty<n<\infty)
$$

repeatedly (see e.g. [1, formula (2.4), p. 17] - the $a_{n}$ there is not the same as our $a_{n}$-or [17, formulas (3.2.1) and (3.2.2), p. 42]; note that $p_{n}$ does not occur on the right-hand side of (7), as the weight function is even), it is possible to express the right-hand side of (6) as a homogeneous polynomial of degree $2 k+1$ of the variables $a_{n+j}$ for certain small values of $j$ (in fact, $|j| \leqslant k$, as we will see).

This polynomial can be described as follows. Imagine an elevator that can travel between floors numbered by integers between $-\infty$ and $+\infty$ which stops at every floor and then continues either in the same direction or in the opposite direction (or stops altogether). Each passage between adjacent floors, briefly called a passage, takes a unit length of time. The length of a trip is the number of passages it consists of. The polynomial in question can be described as the sum of terms corresponding to all trips of length $2 k+1$ from floor 0 to floor -1 in the following way. For such a trip, the corresponding term will be the product of factors $a_{n+j}$ for each passage between floors $j$ and $j-1$ in either direction (i.e. up or down).

It is not difficult to verify that this is indeed a correct description of the polynomial obtained on the right-hand side of (6). In fact, applying (7) repeatedly to the expression $x^{2 k+1} p_{n}(x)$ on the right-hand side of (7), each application decreases the exponent of $x$. After $2 k+1$ applications, the expression obtained will be a linear combination of $p_{n+l}(x)$ for $|l| \leqslant 2 k+1$, with polynomials of $a_{n+j}$ as coefficients. In view of the orthogonality relations, the coefficient of $p_{n-1}(x)$ will be the value of the integral on the right of (6). After $t$ applications of (7) to $x^{2 k+1} p_{n}(x)$, we will have a linear combination of $x^{2 k+1-t} p_{n+l}(x)$ for $|l| \leqslant t$, the coefficient of this polynomial being the sum of terms corresponding, in the way described above, to all trips of length $t$ from floor 0 to floor $l$. This can be easily proved by induction on $t$. The case $t=2 k+1$ and $l=-1$ gives the polynomial described in the preceding paragraph. (The formula expressing $x^{j} p_{n}(x)$ as a linear combination of $p_{n+l}(x)$ for $|l| \leqslant j$, obtained by repeated applications of recurrence equation (7) as described above, is discussed in Nevai [13, Lemma 12, p. 45] in the more general case when $p_{n}(x)$ itself occurs also on the right-hand side of the recurrence equation).

Thus, (6) becomes

$$
n / a_{n}=P\left(a_{n+j}:|j| \leqslant k\right)
$$

where $P$ is the polynomial described above; note that $P$ does not depend on $n$ directly. (Now it is clear why no $a_{n+j}$ for $|j|>k$ occurs on the right-hand side of (8): because the floors $k+1$ or $-k-2$ can not be reached on the trips mentioned in the description of $P$.) (8) can also be written as

$$
n=a_{n} P\left(a_{n+j}:|j| \leqslant k\right)
$$


it will be easy to derive Theorem 1 with the aid of the results of Máté-Nevai [11] from

THEOREM 2. Let $z$ be an arbitrary complex number with $|z|=1$. Then

$$
\sum_{l=-k}^{k} z^{l} \partial\left(x_{0} P\left(x_{j}:|j| \leqslant k\right)\right) / \partial x_{l} \neq 0
$$

holds provided $x_{j}=1$ for $|j| \leqslant k$.

In order to prove this theorem, we will calculate the first order partial derivatives of $P$ at $x_{j}=1,|j| \leqslant k$ :

LEMMA 1. We have

$$
\frac{\partial P\left(x_{j}:|j| \leqslant k\right)}{\partial x_{l}}=\left\{\begin{array}{cc}
2^{2 k+1}-\left(\begin{array}{c}
2 k+1 \\
k
\end{array}\right) & \text { if } l=0 \\
2 \sum_{j=0}^{k-|l|}\left(\begin{array}{c}
2 k+1 \\
j
\end{array}\right) & \text { if } l \neq 0
\end{array}\right.
$$

for $|l| \leqslant k$ and $x_{j}=1(|j| \leqslant k)$.

In light of the description of the polynomial $P$ above, it is easy to give a description of the value of the partial derivative on the left-hand side of (10). It is the number of all passages between floors $l$ and $l-1$ (up or down) summed over all trips of length $2 k+1$ from floor 0 to floor -1 .

We will discuss the following, more general situation. Let $L, h, p, q$ be integers such that $L>0$ and $|p-q|=1$. Let $R_{L, h}(p, q)$ be the number of passages from floor $p$ to floor $q$ (in this direction) summed over all trips of length $L$ from floor 0 to floor $h$. Then

$$
\partial P\left(x_{j}:|j| \leqslant k\right) / \partial x_{l}=R_{2 k+1,-1}(l-1, l)+R_{2 k+1,-1}(l, l-1)
$$

for all $x_{j}=1(|j| \leqslant k)$ according to the description of the left-hand side given in the preceding paragraph. Thus Lemma 1 will be an easy consequence of

LEMMA 2. Let $L, p, q, h$ be integers such that $L>0$ and $|p-q|=1$. In order that $R_{L, h}(p, q)>0$ it is necessary that

$$
L \equiv h \quad(\bmod 2) .
$$

Provided that this is satisfied, we have

$$
R_{L, h}(p, q)=\sum_{j=0}^{d}\left(\begin{array}{l}
L \\
j
\end{array}\right)
$$

(if $d<0$ this sum is taken to be 0 ), where $d$ is defined by the equation

$$
d=d_{L, h}(p, q)=(L-(|p|+1+|h-q|)) / 2 .
$$

Proof. The necessity of (12) for $R_{L, h}(p, q)>0$ is obvious. Assume (12) from now on. It then follows that $d$ as defined by (14) is an integer, since $h$ and

$$
|p|+1+|h-q|=|p|+|q-p|+|h-q|
$$


have the same parity. On account of this equality, (14) can be written as

$$
d=d_{L, h}(p, q)=(L-(|p|+|q-p|+|h-q|)) / 2,
$$

which will be useful later. To prove (13), observe that $R_{L, h}(p, q)$ satisfies the following equations:

$$
\begin{aligned}
& \text { (i) } R_{L, h}(p, q)=R_{L,-h}(-p,-q), \\
& \text { (ii) } R_{L, h}(p, q)=R_{L, 2 p-h}(p, 2 p-q), \\
& \text { (iii) } R_{L, h}(p, q)=R_{L, 2 q-h}(p, q) .
\end{aligned}
$$

Indeed, (i) corresponds to reversing the entire run of the elevator, i.e., for a given trip, of length $L$ from floor 0 to floor $h$, replacing each passage up with a passage down, and each passage down with a passage up. The resulting trip will be of length $L$, will start at floor 0 and stop at floor $-h$. (ii) corresponds to reversing the run of the elevator after it reaches floor $p$ for the first time during its trip, and (iii) corresponds to reversing its run after it reaches floor $q$ the last time during its trip. It is easy to see from (14) or (15) that $d_{L, h}(p, q)$ satisfies the analogous equations. Thus the right-hand side of (13) also satisfies the analogous equations.

Therefore, it is sufficient to establish (13) in case

$$
0 \leqslant p<q \leqslant h .
$$

Indeed, (16)(i) allows one to assume that $p \geqslant 0,(16)(\mathrm{ii})$, that $q>p$, and (16)(iii), that $h \geqslant q$. Assume (17) from now on; then we have

$$
d=(L-h) / 2
$$

according to (15). We can now prove (13) by induction on $L$.

To this end, observe first that (13) is valid in case $L<h$, since both sides are 0 (there is no way to reach floor $h$ from floor 0 in $L$ steps, and the sum on the right of (13) is empty). Assuming $L \geqslant h$ (and (17)), we have

$$
R_{L, h}(p, q)=R_{L-1, h-1}(p, q)+R_{L-1, h+1}(p, q)+\delta_{q h} N_{L-1, h-1},
$$

where $\delta_{q h}=1$ if $q=h$ and 0 otherwise, and $N_{L-1, h-1}$ is the number of all trips from floor 0 to floor $h-1$ in $L-1$ steps, that is (assuming (17)),

$$
N_{L-1, h-1}=\left(\begin{array}{c}
L-1 \\
(L-h) / 2
\end{array}\right)=\left(\begin{array}{c}
L-1 \\
d
\end{array}\right) \text {. }
$$

The first equality here holds in view of the fact that a trip of length $L-1$ from floor 0 to floor $h-1$ consists of $(L+h-2) / 2$ passages up and $(L-h) / 2$ passages down, arranged in an arbitrary order, and the second equality holds in view of (18). Equation (19) is simply saying that the last passage of each trip from floor 0 to floor $h$ is a passage either from floor $h-1$ to floor $h$, or from floor $h+1$ to floor $h$, and the last term adds the number of passages from floor $p$ to $q$ that takes place as the last passage of the trip. This number is $N_{L-1, h-1}$ if $q=h$ (as $p=h-1$ in this case according to (17)), and otherwise it is 0 .

Assume now that $L \geqslant h$ and (13) holds for every quadruple $\left\langle L-1, h^{\prime}, p^{\prime}, q^{\prime}\right\rangle$ replacing $\langle L, h, p, q\rangle$. (The latter is assumed to satisfy (17), but the former is not 
required to satisfy the analogue of (17), as in view of (16) this restriction is not necessary). We distinguish two cases (a) $h>q$, and (b) $h=q$. In both cases,

$$
d_{L-1, h+1}(p, q)=d-1 \quad\left(=d_{L, h}(p, q)-1\right)
$$

and

$$
d_{L-1, h-1}(p, q)= \begin{cases}d & \text { in case }(\mathrm{a}), \\ d-1 & \text { in case }(\mathrm{b}) .\end{cases}
$$

Thus, according to the induction hypothesis, (19) becomes

$$
R_{L, h}(p, q)=\sum_{j=-\infty}^{d}\left(\begin{array}{c}
L-1 \\
j
\end{array}\right)+\sum_{j=-\infty}^{d-1}\left(\begin{array}{c}
L-1 \\
j
\end{array}\right)
$$

in case (a) (we use the convention

$$
\left(\begin{array}{l}
x \\
j
\end{array}\right)=0 \text { for } j<0
$$

thus it does not matter whether the lower limit of the sums is $j=0$ or $j=-\infty$, but the latter choice is technically more convenient), and

$$
R_{L, h}(p, q)=\sum_{j=-\infty}^{d-1}\left(\begin{array}{c}
L-1 \\
j
\end{array}\right)+\sum_{j=-\infty}^{d-1}\left(\begin{array}{c}
L-1 \\
j
\end{array}\right)+\left(\begin{array}{c}
L-1 \\
d
\end{array}\right)
$$

in case (b) (the last term on the right is explained by (20)). Hence (13) follows. This completes the proof of the lemma.

An alternative proof of Lemma 2 is based on

LEMMA 3. The identity

$$
\sum_{j=-\infty}^{\infty}\left(\begin{array}{c}
x+2 j \\
j
\end{array}\right)\left(\begin{array}{c}
y-2 j \\
n-j
\end{array}\right)=\sum_{j=-\infty}^{n}\left(\begin{array}{c}
x+y+1 \\
j
\end{array}\right)
$$

holds for all reals $x$ and $y$ and every integer $n$.

According to (21), the limits of the sums on both sides could just as well be taken to be 0 and $n$. We will give a direct proof of this identity and discuss its relationship to an identity of J. L. W. V. Jensen after the

Alternative Proof of Lemma 2. We will describe another way to derive (13) from (12) and (17); the justification for the assumption of (17) was given in the first proof of Lemma 2.

Assume (12) and (17). We will count the number of passages from floor $p$ to floor $q$ that take place in the time interval $[p+2 j, p+2 j+1]$, where $-\infty<j<\infty$. The number of ways to reach floor $p$ from floor 0 in $p+2 j$ passages is

$$
\left(\begin{array}{c}
p+2 j \\
j
\end{array}\right)
$$

by $p+j$ passages up and $j$ passages down arranged in an arbitrary order (the case $j<0$, while absurd, causes no trouble in view of (21)). The number of ways to reach 
floor $h$ at time $L$, starting at floor $q$ at time $p+2 j+1=q+2 j$ (cf. (17)) is

$$
\left(\begin{array}{c}
L-q-2 j \\
(L-h) / 2-j
\end{array}\right)
$$

by $h-q+(L-h) / 2-j$ passages up and $(L-h) / 2-j$ passages down. (These numbers are integers in view of (12)). It is important to notice that

$$
h-q+(L-h) / 2-j \geqslant(L-h) / 2-j
$$

in view of (17), so the fact that either of these numbers might be negative causes no trouble, since the above binomial coefficient is 0 in this case according to (21) (in the absence of this inequality, it might happen that the left-hand side is negative, while the right-hand side is not; in this case the above binomial coefficient need not be 0 , and this would cause errors in the calculation). The number of passages from floor $p$ to floor $q$ in the above time interval is the product of the above binomial coefficients. Summing for $j$, we obtain (in case (17) holds) that

$$
R_{L, h}(p, q)=\sum_{j=-\infty}^{\infty}\left(\begin{array}{c}
p+2 j \\
j
\end{array}\right)\left(\begin{array}{c}
L-q-2 j \\
(L-h) / 2-j
\end{array}\right)=\sum_{j=-\infty}^{(L-h) / 2}\left(\begin{array}{l}
L \\
j
\end{array}\right),
$$

where the second equality holds according to Lemma 3 (note that $p+(L-q)+1$ $=L$ in view of (17)). In virtue of (18), this is identical to (13) in case (17) holds. Thus the second proof of Lemma 2 is complete.

Proof of Lemma 3. We will outline two ways identity (22) can be obtained. The shorter one is to derive it, from an identity of J. L. W. V. Jensen saying that

$$
\sum_{j=0}^{n}\left(\begin{array}{c}
x+j t \\
j
\end{array}\right)\left(\begin{array}{c}
y-j t \\
n-j
\end{array}\right)=\sum_{j=0}^{n}\left(\begin{array}{c}
x+y-j \\
n-j
\end{array}\right) t^{j}
$$

holds for every complex $x, y$, and $t$, and every integer $n \geqslant 0$ (cf. [7, p. 313; 4; 5, p. 246; 9, Problem 28 in $\S 1.2 .6$ (p. 28; solution p. 485)], plus some papers quoted in [5]). To derive (22) from this, apply the transformation $\left(\begin{array}{l}z \\ l\end{array}\right)=(-1)^{l}\left({ }^{-z+l-1}\right)$ to all three binomial coefficients occurring in (22); the resulting identity will be a special case of (24) with $t=-1$ (cf. (21)).

Another way to prove identity (22) avoids the appeal to (24). To outline this proof, first notice that, both sides of (22) being polynomials of degree $\leqslant n$ in $x$ and $y$, it is sufficient to prove (22) in case $x+y$ is an integer $\geqslant-1$. Writing $I(x, y, n)$ for the formula in (22), it is easy to show by an application of the identity $\left(\begin{array}{c}z-1 \\ j-1\end{array}\right)+\left(\begin{array}{c}z-1 \\ j\end{array}\right)=\left(\begin{array}{c}z \\ j\end{array}\right)$ that $I(x, y-1, n-1)$ and $I(x, y-1, n)$ imply $I(x, y, n)$. Therefore, to complete a proof by induction, we have to show only that $I(x, y, 0)$ and $I(x,-x-1, n)(n>0)$ are valid. The former is obvious, and the latter can be written as

$$
\frac{1}{n !} \sum_{j=0}^{n}\left(\begin{array}{c}
n \\
j
\end{array}\right)(-1)^{n-j} P(j)=1
$$

where $P(j)=\prod_{l=1}^{n}(x+j+l)$. The sum on the left-hand side, however, equals the $n$th difference $(E-1)^{n} P(0)$ of $P(x)$ at $x=0$ (here $E$ is the forward shift operator: $E f(x)=f(x+1))$ according to the binomial theorem. Thus, the left-hand side of 
(25) equals the leading coefficient of $P$; thus (25) is verified (cf. e.g. [8, pp. 131-132] or formula (35) in [9, p. 63]). The proof of Lemma 3 is complete.

Above, we used Lemma 3 to give an alternative proof of Lemma 2. Another way of looking at the two proofs of Lemma 3 is that they establish the second equality in (23) for all integers $L>0$ and $p, q, h$ satisfying (12) and (17) (the first proof giving the third expression in (23) and the second one giving the second expression). As these restrictions allow $n=(L-h) / 2$ to be any integer, and $x=p$ and $y=L-q$ to be any integers $x \geqslant 0$ and $y \geqslant L-h=2 n$, respectively, one obtains a purely combinatorial proof of (22) in this way in all those cases when (22) can be expected to express a combinatorial fact.

We are now in a position to give the

Proof of Lemma 1. Using (11) and (13) one obtains (10). In case $l=0$ in (11), one obtains

$$
\sum_{j=0}^{k-1}\left(\begin{array}{c}
2 k+1 \\
j
\end{array}\right)+\sum_{j=0}^{k}\left(\begin{array}{c}
2 k+1 \\
j
\end{array}\right)
$$

but this is easily seen to be equal to the expression given on the right-hand side of (10). In case $l \neq 0$, one obtains the right-hand side of (10) directly. The proof of Lemma 1 is complete.

Theorem 1 will be a simple corollary of

LEMMA 4. Let $\lambda_{l}, 0 \leqslant l \leqslant k+2(k \geqslant 0)$, be a strictly convex sequence of real numbers, i.e. such that

$$
\Delta^{2} \lambda_{l}=\lambda_{l}-2 \lambda_{l+1}+\lambda_{l+2}>0 \quad(0 \leqslant l \leqslant k)
$$

( $\Delta$ is the forward difference operator, i.e. $\Delta z_{l}=z_{l+1}-z_{l}$ ), and assume

$$
\lambda_{k+1}=\lambda_{k+2}=0 \text {. }
$$

Then the equation

$$
\lambda_{0}+\sum_{l=1}^{k} \lambda_{l}\left(z^{l}+z^{-l}\right)=0
$$

has no roots of absolute value 1.

Proof. Assuming $|z|=1$, we may write $z=e^{i x}$ for real $x$. Then $z^{l}+z^{-l}=2 \cos l x$; dividing equation (27) by 2 and performing summation by parts (i.e. Abel rearrangement) on the left-hand side twice, we obtain

$$
\sum_{l=0}^{k}(l+1) K_{l}(x) \Delta^{2} \lambda_{l}=0
$$

where $K_{l}$ is the $l$ th Fejér kernel, i.e.

$$
(l+1) K_{l}(x)=\sum_{j=0}^{l}\left(1 / 2+\sum_{r=1}^{l} \cos r x\right)=(1 / 2)(\sin ((l+1) x / 2) / \sin (x / 2))^{2}
$$

(interpret the right-hand side as its limit at points where the denominator is 0 ; $\mathrm{cf}$. e.g. [18, Vol. I, p. 88]). It is seen from here that $K_{l}(x) \geqslant 0$; moreover, $K_{0}(x)=1 / 2$ $(>0)$ for every $x$. Thus (28) is impossible in view of (26). The proof is complete. 
Note that only $\Delta^{2} \lambda_{l} \geqslant 0$ was used in most cases instead of the full force of (26). We need to assume strict inequality either only in case $l=0$ or else, for example, in case $l=r$ and $l=s$ for some $r, s$ with $0<r<s \leqslant k$ that are relatively prime (this latter is sufficient, since, as is easily seen, $K_{r}(x)=K_{s}(x)=0$ cannot happen for any $x)$.

We are now able to present the

Proof of Theorem 2. Observe that we have

$$
P(1:|j| \leqslant k)=\left(\begin{array}{c}
2 k+1 \\
k
\end{array}\right)
$$

for the polynomial $P$ discussed in (8)-(10). Indeed, for $x_{j}=1(|j| \leqslant k)$ all terms of $P$ being 1 , this is just the number of terms in $P$, i.e. the number of trips of length $2 k+1$ from floor 0 to floor -1 ( $k$ passages up and $k+1$ passages down, arranged in an arbitrary order). Thus, in view of (10) (and the product rule for differentiation), (9) becomes

$$
\lambda_{0}+\sum_{l=1}^{k} \lambda_{l}\left(z^{l}+z^{-l}\right) \neq 0
$$

where

$$
\lambda_{0}=2^{2 k+1}=2 \sum_{j=-\infty}^{k}\left(\begin{array}{c}
2 k+1 \\
j
\end{array}\right)
$$

(cf. (21)) and

$$
\lambda_{l}=2 \sum_{j=-\infty}^{k-l}\left(\begin{array}{c}
2 k+1 \\
j
\end{array}\right) \quad(0<l \leqslant k) .
$$

Comparison with the former formula shows that the latter formula is valid even in case $l=0$. Putting $\lambda_{k+1}=\lambda_{k+2}=0$ (which, again, is in harmony with the latter formula), it is seen that

$$
\Delta^{2} \lambda_{l}=2\left(\begin{array}{c}
2 k+1 \\
k-l
\end{array}\right)-2\left(\begin{array}{c}
2 k+1 \\
k-(l+1)
\end{array}\right)>0 \quad(0 \leqslant l \leqslant k) .
$$

Therefore Lemma 4 shows that (29) must hold for $|z|=1$. This establishes (9), completing the proof of Theorem 2.

Finally, we will show how Theorem 2 and the results of Máté-Nevai [11] can be put together to give the

Proof of TheOrem 1. Writing

$$
y_{n}=\left(\begin{array}{c}
2 k+1 \\
k
\end{array}\right)^{-1 /(2 k+2)} a_{n} n^{-1 /(2 k+2)},
$$

we have

$$
\lim _{n \rightarrow \infty} y_{n}=1
$$

in view of (1) being valid with $\beta=2 k+2$ (cf. (5)). Putting

$$
F\left(x_{j}:|j| \leqslant k\right)=x_{0} P\left(x_{j}:|j| \leqslant k\right) \text {, }
$$


(8) becomes

$$
F\left(y_{n+j}(1+j / n)^{1 /(2 k+2)}:|j| \leqslant k\right)=1,
$$

that is

$$
H\left(y_{n-j}, \ldots, y_{n+j}, 1 / n\right)=0
$$

with

$$
H\left(x_{j}:-k \leqslant j \leqslant k+1\right)=F\left(x_{j}\left(1+j x_{k+1}\right)^{1 / 12 k+2)}:|j| \leqslant k\right) .
$$

Now, according to the main theorem proved in Máté-Nevai [11], (30), (31), and the condition that

$$
\sum_{l=-k}^{k} z^{l} \partial H\left(x_{j}:-k \leqslant j \leqslant k+1\right) / \partial x_{j} \neq 0
$$

for $x_{j}=1(|j| \leqslant k), x_{k+1}=0$, and for any $z$ with $|z|=1$ ensure that $y_{n}$ has an asymptotic expansion

$$
y_{n} \sim \sum_{l=0}^{\infty} c_{l} n^{-l},
$$

provided that $H$ is a $C^{\infty}$ function in a neighborhood of the point $x_{j}=1(|j| \leqslant k)$, $x_{k+1}=0$. Given that (32) is satisfied, since it is equivalent to (9), the existence of expansion (33) follows. (The quoted theorem in [11] gives the expansion of $y_{n-k}$ in terms of $n^{-l}$, i.e. the expansion of $y_{n}$ is obtained in terms of

$$
(n+k)^{-l}=n^{-l}(1+k / n)^{-l} \text {. }
$$

Expansion (33) can then be derived by using the binomial expansion for $(1+k / n)^{-l}$; cf. the remark after (27) in [11].) One can show that

$$
c_{l}=0 \text { for odd } l
$$

in exactly the same way as (28) of [11] was proved in that paper, by using the obvious relation

$$
F\left(x_{j}:|j| \leqslant k\right)=F\left(x_{-j}:|j| \leqslant k\right) .
$$

The proof of Theorem 1 is complete.

\section{REFERENCES}

1. G. Freud, Orthogonal polynomials, Pergamon Press, Oxford and New York, 1966.

2. __ On the greatest zero of an orthogonal polynomial, Acta Sci. Math. Szeged 24 (1973), 91-97.

3. On the coefficients in the recursion formulae of orthogonal polynomials, Proc. Roy. Irish Acad. Sect. A 76 (1976), 1-6.

4. H. W. Gould, Generalization of a theorem of Jensen concerning convolutions, Duke Math. J. 27 (1960), 71-76.

5. H. W. Gould and J. Kauchy, Evaluation of a class of binomial coefficient summations, J. Combin. Theory 1 (1966), 233-247.

6. P. Henrici, Applied and computational complex analysis, Vol. 2, Wiley, New York and London, 1977.

7. J. L. W. V. Jensen, Sur une identité d'Abel et sur d' autres formules analogues, Acta Math. 26 (1902), $307-318$.

8. C. Jordan, Calculus of finite differences, 3rd ed., Chelsea, New York, 1965. 
9. D. E. Knuth, The art of computer programming, Vol. 1, 2nd ed., Addison-Wesley, Reading, Mass., 1973.

10. J. S. Lew and D. A. Quarles, Jr., Nonnegative solutions of a nonlinear recurrence, J. Approx. Theory 38 (1983), 357-379.

11. A. Máté and P. Nevai, Asymptotics for solutions of smooth recurrence equations, Proc. Amer. Math. Soc. (to appear).

12. H. N. Mhaskar and E. B. Saff, Extremal problems for polynomials with exponential weights, Trans. Amer. Math. Soc. 285 (1984), 203-234.

13. P. Nevai, Orthogonal polynomials, Mem. Amer. Math. Soc., Vol. 18 (1979), No. 213.

14. Orthogonal polynomials associated with $\exp \left(x^{-4}\right)$, Canadian Math. Soc. Conference Proc. 3 (1983), 263-285.

15. __ Asymptotics for orthogonal polynomials associated with $\exp \left(x^{-4}\right)$, SIAM J. Math. Anal. 15 (1984).

16. E. A. Rahmanov, On asymptotic properties of polynomials orthogonal on the real axis, Math. Sb. (N.S.) 119 (161) (1982), 163-203. (In Russian)

17. G. Szegö, Orthogonal polynomials, 4th ed., Amer. Math. Soc. Colloq. Publ., Vol. 23, Amer. Math. Soc., Providence, R.I., 1978.

18. A. Zygmund, Trigonometric series, Vols. I and II, 2nd ed., Cambridge Univ. Press, Cambridge and New York, 1977.

19. Al. Magnus, A proof of Freud's conjecture about the orthogonal polynomials related to $|x|^{\rho} \exp \left(-x^{2 m}\right)$ for integer $m$ (manuscript).

Department of Mathematics, Brooklyn College of the City University of New York, Bedford Avenue and Avenue H, Brooklyn, New York 11210 (Current address of Attila Máté)

Department of Mathematics, Ohio State University, Columbus, Ohio 43210 (Current address of Paul Nevai and Thomas Zaslavsky) 